\title{
RÉSOLVANT GÉNÉRALISÉ ET SÉPARATION DES POINTS SINGULIERS QUASI-FREDHOLM
}

\author{
J. PH. LABROUSSE ET M. MBEKHTA
}

\begin{abstract}
Resumé. C. Apostol et K. Clancey (Trans. Amer. Math. Soc. 215 (1976), 293-300), ont introduit la notion de "projection spectrale généralisée". Cette notion permet, en particulier, de séparer les ensembles finis de points singuliers dans le domaine semi-Fredholm $\left(\rho_{s \phi}(A)\right)$ d'un opérateur $A$ borné dans un Hilbert $H$. Dans ce travail, on se propose de généraliser ce résultat au domaine quasi-Fredholm de $A\left(\rho_{q \phi}(A)\right)$, et pour cela, nous donnons une nouvelle représentation triangulaire du type d'Apostol. D'autre part on construit, pour un opérateur fermé à domaine dense dans $H$, un résolvant généralisé vérifiant l'identité de la résolvante et analytique dans le domaine régulier de Fredholm de $A\left(\rho_{\phi}^{r}(A)\right)$ sauf éventuellement sur un ensemble au plus dénombrable de points situés prés de la frontière de $\rho_{\phi}(A)$.
\end{abstract}

\section{Préliminaires}

Soit $H$ un espace de Hilbert et $A$ un opérateur fermé de domaine $D(A)$ et d'image $R(A)$ dans $H$. On note $N(A), \sigma(A)$ et $\rho(A)$ respectivement le noyau, le spectre et l'ensemble résolvant de $A$.

Soit $\mathscr{U}$ une partie de $\mathbb{C}$; on dira que $A$ admet un opérateur résolvant (ou inverse) généralisé $\operatorname{Rg}(A, \lambda)$ dans $\mathscr{U}$ si $\forall \lambda \in \mathscr{U}, \operatorname{Rg}(A, \lambda)$ est un opérateur borné de $H$ dans $D(A)$ tel que:

$$
(A-\lambda I) \operatorname{Rg}(A, \lambda)(A-\lambda I)=(A-\lambda I) \quad \text { et } \quad \operatorname{Rg}(A, \lambda)(A-\lambda I) \operatorname{Rg}(A, \lambda)=\operatorname{Rg}(A, \lambda) .
$$

Remarques. (1) En général $\operatorname{Rg}(A, \lambda)$ n'est pas unique. Si toutefois $\lambda \in \rho(A)$, alors $\operatorname{Rg}(A, \lambda)=(A-\lambda I)^{-1}$ l'opérateur résolvant habituel.

(2) Si on note $P_{\lambda}=(A-\lambda I) \operatorname{Rg}(A, \lambda)$ et $Q_{\lambda}=\operatorname{Rg}(A, \lambda)(A-\lambda I)$ alors $P_{\lambda}$ et $Q_{\lambda}$ sont des projections telles que $R\left(P_{\lambda}\right)=R(A-\lambda I)$ et $N\left(Q_{\lambda}\right)=N(A-\lambda I)$.

On dira que $\operatorname{Rg}(A, \lambda)$ vérifie l'identité de la résolvante dans $\mathscr{U}$ si $\forall \lambda, \mu$ appartenant à une même composante connexe de $\mathscr{U}$

$$
\operatorname{Rg}(A, \lambda)-\operatorname{Rg}(A, \mu)=(\lambda-\mu) \operatorname{Rg}(A, \lambda) \operatorname{Rg}(A, \mu) .
$$

Dans $[7,9,10$ et 11$]$ on trouve une généralisation de $\rho(A)$ où la notion d'inverse est remplacée par celle d'inverse généralisé. Notons $\operatorname{reg}(A)$ l'ensemble

Received by the editors November 13, 1989 and, in revised form, June 5, 1990.

1980 Mathematics Subject Classification (1985 Revision). Primary 47A53, 47B05.

Key words and phrases. Opérateurs de Fredholm, quasi-Fredholm, résolvant généralisé, spectre généralisé. 
régulier (ou l'ensemble résolvant généralisé) de $A$, défini par:

$\operatorname{reg}(A)=\{\lambda \in \mathbb{C} ; A$ admet un opérateur résolvant généralisé

$$
\text { analytique dans un voisinage } \mathscr{U} \text { de } \lambda\} \text {. }
$$

Il est clair que $\operatorname{reg}(A)$ est ouvert et $\rho(A) \subseteq \operatorname{reg}(A)$. Le spectre généralisé $\sigma_{g}(A)$ (complément de $\operatorname{reg}(A)$ dảns $\mathbb{C}$ ), possède des propriétés analogues à celles de $\sigma(A)$ dans la théorie spectrale classique (cf. [10, 11]).

Enfin, on dira que $A$ est régulier si $R(A)$ est fermé et $\forall n \geq 0, N\left(A^{n}\right) \subseteq$ $R(A)$.

Théorème 1.1. Soit $A$ un opérateur fermé avec $D(A)$ dense alors les conditions suivantes sont équivalentes:

(i) $\lambda_{0} \in \operatorname{reg}(A)$,

(ii) $A-\lambda_{0} I$ est régulier,

(iii) $R\left(A-\lambda_{0} I\right)$ est fermé dans $H$ et l'application $\lambda \mapsto P_{R(A-\lambda I)}$ est continue en $\lambda_{0}$.

(iv) $R\left(A-\lambda_{0} I\right)$ fermé dans $H$ et l'application $\lambda \mapsto P_{N(A-\lambda I)}$ est continue en $\lambda_{0}$ (où $P_{M}$ est la projection orthogonale sur $M$ ).

(v) $c\left(A-\lambda_{0} I\right)>0$ et l'application $\lambda \mapsto c(A-\lambda I)$ est continue en $\lambda_{0}$, où $c(A)$ est la conorme de $A$ définie par

$$
c(A)=\inf \left\{\|A u\| ; u \in D(A) \cap N(A)^{\perp} \text { et }\|u\|=1\right\} \quad(c f .[6])
$$

(vi) $\bar{\lambda}_{0} \in \operatorname{reg}\left(A^{*}\right)$.

Démonstration. (i) $\Leftrightarrow$ (ii) Voir [9, Théorème 2.6].

(i) $\Leftrightarrow$ (iii) $\Leftrightarrow$ (iv) $\Leftrightarrow$ (v) Voir [11, Théorème 2.1].

(i) $\Leftrightarrow$ (vi) Voir [7, Corollaire 4.1.2].

On aura besoin aussi de la notion de coeur d'un opérateur qu'on note $\operatorname{Co}(A)$ par définition c'est le plus grand sous-espace $M$ de $H$ tel que $A(M \cap D(A))=M$ (sur l'existence de $\operatorname{Co}(A)$ cf. [12]).

Proposition 1.2 [8]. Si $0 \in \operatorname{reg}(A)$ alors

(i) $\operatorname{Co}(A-\lambda I)$ est constant dans la composante connexe $\Omega_{0}$ de $\operatorname{reg}(A)$ contenant zéro. En plus on a

$$
\operatorname{Co}(A)=\bigcap_{n \geq 0} R\left(A^{n}\right)=\bigcap_{j \geq 0} R\left(A-\lambda_{j} I\right)
$$

où $\left\{\lambda_{j}\right\}$ est une suite de points, tous distincts, de $\Omega_{0}$ qui converge vers zéro.

(ii) $H_{0}(A) \subseteq \mathrm{Co}(A)=H_{0}\left(A^{*}\right)^{\perp}$ où $H_{0}(A)=\left\{u \in D\left(A^{\infty}\right) ; \quad \lim _{n \rightarrow \infty}\left\|A^{n} u\right\|^{1 / n}\right.$ $=0\}$.

Corollaire 1.3. Soit $A$ un opérateur fermé avec $D(A)$ dense dans $H$ et tel que $0 \in \operatorname{reg}(A)$ alors

$$
\text { si } \operatorname{codim}(\operatorname{Co}(A))=m<\infty \text { alors } m=0 \text {. }
$$

Démonstration. Supposons que $\operatorname{codim}(\operatorname{Co}(A))=m$; alors $m=\operatorname{dim}\left(\operatorname{Co}(A)^{\perp}\right) \geq$ $\operatorname{dim}\left(H_{0}\left(A^{*}\right)\right)$. La dimension de $H_{0}\left(A^{*}\right)$ étant finie, $H_{0}\left(A^{*}\right)$ est fermé et comme $A^{*}$ est régulier on en déduit que $H_{0}\left(A^{*}\right)=\{0\}$ (cf. [9, Théorème 2.11]) et par conséquent que $R(A)=H=\operatorname{Co}(A)$ ce qui montre que $m=0$.

Proposition 1.4. Soit $A$ un opérateur fermé avec $D(A)$ dense dans $H$ et $\mathscr{U}$ un ouvert connexe de $\operatorname{reg}(A)$, alors les conditions suivantes sont équivalentes 
(a) Il existe $E, F$ sous-espaces fermés de $H$ tels que

$$
\forall \lambda \in \mathscr{U} \quad R(A-\lambda I) \oplus F=N(A-\lambda I) \oplus E=H .
$$

(b) $A$ admet un résolvant généralisé analytique et vérifiant l'identité de la résolvante dans $\mathscr{U}$.

Démonstration. (a) $\Rightarrow$ (b) : on a $\forall \lambda \in \mathscr{U}, R(A-\lambda I) \oplus F=N(A-\lambda I) \oplus E=H$. Soit $P_{\lambda}$ la projection sur $R(A-\lambda I)$ correspondant à la première décomposition et $Q_{\lambda}$ la projection sur $E$ correspondant à la deuxième décomposition.

Soit maintenant $u \in H$, alors $P_{\lambda} u \in R(A-\lambda I)$ et il existe $v \in D(A)$ tel que $(A-\lambda I) v=P_{\lambda} u$.

On posera par définition $\operatorname{Rg}(A, \lambda) u=Q_{\lambda} v \in D(A)$. Montrons que $\operatorname{Rg}(A, \lambda)$ est ainsi bien défini. En effet soit $w \in H$ tel que $(A-\lambda I) w=P_{\lambda} u$. Alors $v-w \in N(A-\lambda I)$ d'où $Q_{\lambda}(v-w)=0$ ou encore $Q_{\lambda} v=Q_{\lambda} w$ ce qui montre que $\operatorname{Rg}(A, \lambda)$ ne dépend pas du choix de $v$.

D'autre part on a $\forall v \in D(A) \quad\left(I-Q_{\lambda}\right) v \in N(A-\lambda I)$ et $(A-\lambda I)\left(I-Q_{\lambda}\right) v=0$ donc

$$
(A-\lambda I) v=(A-\lambda I) Q_{\lambda} v
$$

Posons $(A-\lambda I) v=u=P_{\lambda} u$; alors $\operatorname{Rg}(A, \lambda)(A-\lambda I) v=\operatorname{Rg}(A, \lambda) u=Q_{\lambda} v$. Donc

$$
\operatorname{Rg}(A, \lambda)(A-\lambda I)=Q_{\lambda} \quad \text { sur } D(A) .
$$

Si $u \in H$ avec $P_{\lambda} u=(A-\lambda I) v$ alors $(A-\lambda I) \operatorname{Rg}(A, \lambda) u=(A-\lambda I) Q_{\lambda} v=$ $(A-\lambda I) v=P_{\lambda} u$. Donc

$$
(A-\lambda I) \operatorname{Rg}(A, \lambda)=P_{\lambda} \quad \text { sur } H .
$$

Montrons que $\operatorname{Rg}(A, \lambda)$ est un résolvant généralisé de $A$ dans $\mathscr{U}$. Par défini-

tion, $\operatorname{Rg}(A, \lambda)$ est à valeurs dans $D(A)$. Vérifions que

$$
(A-\lambda I) \operatorname{Rg}(A, \lambda)(A-\lambda I)=(A-\lambda I) \quad \text { sur } D(A) .
$$

En utilisant (1) et la définition de $\operatorname{Rg}(A, \lambda)$ on a $\forall v \in D(A)$,

$$
(A-\lambda I) \operatorname{Rg}(A, \lambda)(A-\lambda I) v=(A-\lambda I) Q_{\lambda} v=(A-\lambda I) v
$$

et donc (4) est démontré.

Vérifions maintenant que

$$
\operatorname{Rg}(A, \lambda)(A-\lambda I) \operatorname{Rg}(A, \lambda)=\operatorname{Rg}(A, \lambda) \text { sur } H .
$$

Soit $u \in H$ avec $P_{\lambda} u=(A-\lambda I) v$, alors par définition de $\operatorname{Rg}(A, \lambda)$ on a $\operatorname{Rg}(A, \lambda) u=Q_{\lambda} v$, et en appliquant (1) et (2) on obtient d'une part que $(A-\lambda I) \operatorname{Rg}(A, \lambda) u=(A-\lambda I) Q_{\lambda} v=(A-\lambda I) v$ et d'autre part, $\operatorname{Rg}(A, \lambda)(A-\lambda I) \operatorname{Rg}(A, \lambda) u=\operatorname{Rg}(A, \lambda)(A-\lambda I) v=Q_{\lambda} v=\operatorname{Rg}(A, \lambda) u$ et $(5)$ est démontré.

Finalement soit $u \in H$ et $C$ un voisinage compact de $\lambda$ dans $\mathscr{U} . P_{\lambda} u \in$ $R(A-\lambda I)$ alors $\exists v \in D(A) \cap N(A-\lambda I)^{\perp}$ tel que $\left\|P_{\lambda} u\right\|=\|(A-\lambda I) v\| \geq$ $c(A-\lambda I)\|v\|$. Donc

$$
\begin{aligned}
\|\operatorname{Rg}(A, \lambda) u\| & =\left\|Q_{\lambda} v\right\| \leq\left\|Q_{\lambda}\right\|\|v\| \leq\left\|Q_{\lambda}\right\|[c(A-\lambda I)]^{-1}\left\|P_{\lambda} u\right\| \\
& \leq\left\|Q_{\lambda}\right\|\left\|P_{\lambda}\right\|[c(A-\lambda I)]^{-1}\|u\| .
\end{aligned}
$$


L'application $\lambda \mapsto c(A-\lambda I)$ est continue et strictement positive dans $\operatorname{reg}(A)$ (cf. Théorème 1.1(v)). Comme $C \subseteq \operatorname{reg}(A)$ est compact, on déduit que l'application $\lambda \mapsto[c(A-\lambda I)]^{-1}$ admet un maximum dans $C$. De même les applications $\lambda \mapsto$ $Q_{\lambda}$ et $\lambda \mapsto P_{\lambda}$ sont continues dans $\operatorname{reg}(A)$, et on en déduit qu'il existe $M>0$ tel que $\forall \lambda \in C,\|\operatorname{Rg}(A, \lambda) u\| \leq M\|u\|$. Donc $\operatorname{Rg}(A, \lambda)$ est un opérateur continu.

Montrons maintenant que $\operatorname{Rg}(A, \lambda)$ vérifie l'identité de la résolvante dans $\mathscr{U}$. Remarquons d'abord que si $\lambda, \mu \in \mathscr{U}$ alors $\left(I-P_{\mu}\right)\left(I-P_{\lambda}\right)=\left(I-P_{\lambda}\right)$ (d'où $P_{\mu} P_{\lambda}=P_{\mu}$ ) et $Q_{\mu} Q_{\lambda}=Q_{\lambda}$.

Soit $u \in H$ et $\lambda, \mu \in \mathscr{U}$, en utilisant (2) et (3) on a:

$$
\begin{aligned}
& \operatorname{Rg}(A, \lambda) u-\operatorname{Rg}(A, \mu) u=Q_{\mu} \operatorname{Rg}(A, \lambda) u-\operatorname{Rg}(A, \mu) P_{\lambda} u \\
& \quad=\operatorname{Rg}(A, \mu)(A-\mu I) \operatorname{Rg}(A, \lambda) u-\operatorname{Rg}(A, \mu)(A-\lambda I) \operatorname{Rg}(A, \lambda) u \\
& \quad=(\lambda-\mu) \operatorname{Rg}(A, \mu) \operatorname{Rg}(A, \lambda) u .
\end{aligned}
$$

D’où

$$
\operatorname{Rg}(A, \lambda)-\operatorname{Rg}(A, \mu)=(\lambda-\mu) \operatorname{Rg}(A, \mu) \operatorname{Rg}(A, \lambda) .
$$

En passant à la limite pour $\mu \rightarrow \lambda$ on voit que $\operatorname{Rg}(A, \lambda)$ est continue en $\lambda$. Donc en divisant les deux termes de l'égalité ci-dessus par $\lambda-\mu$ et en passant à la limite pour $\mu \rightarrow \lambda$ on trouve:

$$
(\operatorname{Rg}(A, \lambda))^{\prime}=(\operatorname{Rg}(A, \lambda))^{2}
$$

formule qui généralise la formule bien connue pour la dérivée de l'opérateur résolvant habituel et qui établit que $\operatorname{Rg}(A, \lambda)$ est analytique dans $\mathscr{U}$.

(b) $\Rightarrow($ a) : On a $\forall \lambda, \mu \in \mathscr{U}$,

$$
\operatorname{Rg}(A, \lambda)-\operatorname{Rg}(A, \mu)=(\lambda-\mu) \operatorname{Rg}(A, \lambda) \operatorname{Rg}(A, \mu)
$$

et

$$
\operatorname{Rg}(A, \mu)-\operatorname{Rg}(A, \lambda)=(\mu-\lambda) \operatorname{Rg}(A, \mu) \operatorname{Rg}(A, \lambda)
$$

D'où

$$
-(\operatorname{Rg}(A, \lambda)-\operatorname{Rg}(A, \mu))=-(\lambda-\mu) \operatorname{Rg}(A, \mu) \operatorname{Rg}(A, \lambda) .
$$

De la première et la dernière égalité on déduit que

$$
\operatorname{Rg}(A, \lambda) \operatorname{Rg}(A, \mu)=\operatorname{Rg}(A, \mu) \operatorname{Rg}(A, \lambda) .
$$

Montrons que le noyau de $\operatorname{Rg}(A, \lambda))$ est constant par rapport à $\lambda$. En effet, soit $\lambda, \mu \in \mathscr{U}$ et $u \in N(\operatorname{Rg}(A, \lambda))$ alors

$$
\operatorname{Rg}(A, \mu) u=-(\lambda-\mu) \operatorname{Rg}(A, \mu) \operatorname{Rg}(A, \lambda) u+\operatorname{Rg}(A, \lambda) u=0
$$

donc $N(\operatorname{Rg}(A, \lambda)) \subseteq N(\operatorname{Rg}(A, \mu))$. En interchangeant $\lambda$ et $\mu$ on obtient l'autre inclusion et par conséquent $N(\operatorname{Rg}(A, \lambda))=N(\operatorname{Rg}(A, \mu))$. On notera, $E$ la valeur commune à tous les noyaux de $\operatorname{Rg}(A, \lambda)$ pour $\lambda \in \mathscr{U}$.

Montrons que l'image de $\operatorname{Rg}(A, \lambda)$ est constante par rapport à $\lambda$. En effet, (6) et (7) impliquent que $\operatorname{Rg}(A, \lambda)=(\lambda-\mu) \operatorname{Rg}(A, \mu) \operatorname{Rg}(A, \lambda)+\operatorname{Rg}(A, \mu)=$ $\operatorname{Rg}(A, \mu)[(\lambda-\mu) \operatorname{Rg}(A, \lambda)+I)]$. Ceci montre que l'image de $\operatorname{Rg}(A, \lambda)$ est inclue dans l'image de $\operatorname{Rg}(A, \mu)$. En interchangeant $\lambda$ et $\mu$ on obtient l'autre inclusion. Soit $F$ la valeur commune de toutes les adhérences des images de $\operatorname{Rg}(A, \lambda)$ pour $\lambda \in \mathscr{U}$.

Soit $u \in H$ alors $u=[I-(A-\lambda I) \operatorname{Rg}(A, \lambda)] u+(A-\lambda I) \operatorname{Rg}(A, \lambda) u$ d'où $u \in N(\operatorname{Rg}(A, \lambda))+R(A-\lambda I)=E+R(A-\lambda I)$ donc $H \subseteq E+R(A-\lambda I)$. Soit 
maintenant $u \in N(\operatorname{Rg}(A, \lambda)) \cap R(A-\lambda I)$ alors $u=(A-\lambda I) v$ et $\operatorname{Rg}(A, \lambda) u=$ $\operatorname{Rg}(A, \lambda)(A-\lambda I) v=0$ donc $(A-\lambda I) \operatorname{Rg}(A, \lambda)(A-\lambda I) v=0$ et par conséquent $u=(A-\lambda I) v=0$. Donc

$$
H=R(A-\lambda I) \oplus N(\operatorname{Rg}(A, \lambda))=R(A-\lambda I) \oplus E .
$$

Finalement, comme $\operatorname{Rg}\left(A^{*}, \bar{\lambda}\right)=\operatorname{Rg}(A, \lambda)^{*}$ on voit que $A^{*}$ satisfait les mêmes hypothèses que $A$ et par conséquent que $\lambda \in \mathscr{U}$ implique que

$H=R\left(A^{*}-\overline{\lambda I}\right) \oplus N\left(\operatorname{Rg}\left(A^{*}, \bar{\lambda}\right)\right)=R\left(A^{*}-\overline{\lambda I}\right)^{\perp} \oplus N\left(\operatorname{Rg}\left(A^{*}, \bar{\lambda}\right)\right)^{\perp}=N(A-\lambda I) \oplus F$ oú $F=N\left(\operatorname{Rg}\left(A^{*}, \bar{\lambda}\right)\right)^{\perp}=R(\operatorname{Rg}(A, \lambda))$ ne dépend pas de $\lambda$.

\section{Sur le RÉSOlVANT GÉNÉRALISÉ DANS LE dOMAINE DE Fredholm}

Dans ce paragraphe, on notera $\rho_{\phi}(A)$ l'ensemble résolvant de Fredholm, défini par

$$
\rho_{\phi}(A)=\{\lambda \in \mathbb{C} ; A-\lambda I \text { est un opérateur de Fredholm }\} .
$$

Notons aussi:

$$
\rho_{\phi}^{r}(A)=\rho_{\phi}(A) \cap \operatorname{reg}(A), \quad \rho_{\phi}^{s}(A)=\rho_{\phi}(A) \backslash \rho_{\phi}^{r}(A) .
$$

Remarque. $\rho_{\phi}^{s}(A)$ est dénombrable et n'admet pas de points d'accumulation dans $\rho_{\phi}(A)$ (cf. par exemple [7, Proposition 4.3.1(a)]).

Théorème 2.1. Soit $A$ un opérateur fermé avec $D(A)$ dense dans $H$ et $\Omega$ une composante connexe de $\rho_{\phi}^{r}(A)$. Alors pour tout compact $K$ de $\Omega$ il existe un résolvant généralisé de $A$ vérifiant l'identité de la résolvante et analytique dans K.

Remarque. Pour la démonstration de ce Théorème on aura besoin de quelques résultats préliminaires et sans perte de généralité on supposera que $0 \in K$.

En utilisant la Proposition 1.2(i), il est facile de voir que:

$$
\operatorname{Co}(A)=\bigcap_{\left\{\lambda_{i}\right\} \subset K} R\left(A-\lambda_{i} I\right) \quad \text { où }\left\{\lambda_{i}\right\} \text { est une suite infinie de points distincts. }
$$

Lemme 2.2. Si $\operatorname{Co}(A) \neq H$ alors $\exists f_{A} \in D(A)$ tel que $\forall \lambda \in K, f_{A} \notin R(A-\lambda I)$. Quand aucune confusion n'est possible on notera $f_{A}$ simplement $f$.

Démonstration. Comme $\operatorname{Co}(A) \neq H$ et $D(A)$ est dense dans $H$, on en déduit que $\exists u \in D(A)$ et $u \notin \operatorname{Co}(A)$ avec $u \neq 0$.

Posons

$$
\Delta=\left\{\lambda \in K ; \exists n(\lambda) \geq 1, u \in R(A-\lambda I)^{n(\lambda)} \text { et } u \notin R(A-\lambda I)^{n(\lambda)+1}\right\} .
$$

Si $\Delta=\varnothing$ alors on prend $f=u$. Supposons que $\Delta \neq \varnothing$ et montrons que $\Delta$ est fini. Si $\Delta$ est infini alors il existe $\left\{\lambda_{i}\right\} \subset \Delta \subset K$, une suite infinie et d'après la remarque précédente on en déduit que $u \in \operatorname{Co}(A)$ ce qui contredit le fait que $u \notin \operatorname{Co}(A)$. Donc si $u \in \bigcap_{\lambda \in \Delta} R(A-\lambda I)^{n(\lambda)}, \exists w \in D(A)$ tel que $u=\prod_{\lambda \in \Delta}(A-\lambda I)^{n(\lambda)} w$ et $\forall \lambda \in K, w \notin R(A-\lambda I)$. Il suffit maintenant de prendre $f=w$.

Remarque. $\forall \lambda \in K, R(A-\lambda I) \oplus\{f\}$ est fermé, où $\{f\}$ désigne l'espace engendré par $f$. 
Lemme 2.3. Posons

$$
C_{1}=\bigcap_{\lambda \in K \backslash\{0\}}(R(A-\lambda I) \oplus\{f\}) \quad \text { et } \quad C_{2}=\bigcap_{\lambda \in K}(R(A-\lambda I) \oplus\{f\}) .
$$

(i) $C_{1}=C_{2}$ (et tous deux seront notés $C$ ),

(ii) $\forall u \in D(A): u \in C_{1} \Leftrightarrow A u \in C_{1}$.

Démonstration. (i) Evidemment il suffit de montrer que $C_{1} \subseteq C_{2}$. Soit $u \in C_{1}$ et $\left\{\lambda_{i}\right\} \subset K$ une suite qui converge vers $0 . \forall i \in \mathbb{N}$, soit $P_{i}$ la projection orthogonale sur $R\left(A-\lambda_{i} I\right)+\{f\}$ et $P_{0}$ la projection orthogonale sur $R(A)+\{f\}$. Alors, d'après le Corollaire 1.3.5 de [7], (en prenant $N^{\perp}=\{f\}, M=R(A)$, $\left.M^{\prime}=R\left(A-\lambda_{i} I\right)\right)$ et le Théorème 1.1(iii), $\lim _{i \rightarrow \infty} P_{i}=P_{0}$. Donc comme $\forall i \in \mathbb{N}, u=P_{i} u$ on $a: u=\lim _{i \rightarrow \infty} P_{i} u=P_{0} u$ d'où $u \in C_{2}$.

(ii) $(\Rightarrow)$. Soit $u \in C$. Alors $\forall \lambda \in K \quad u=(A-\lambda I) v+\alpha f$. Comme $u$ et $f \in D(A)$ alors $(A-\lambda I) v \in D(A)$ d'où $v \in D\left(A^{2}\right)$. Donc $A u=(A-\lambda I) A v+$ $\alpha A f=(A-\lambda I)(A v+\alpha f)+\alpha \lambda f \in R(A-\lambda I) \oplus\{f\}$ pour tout $\lambda$ dans $K$, et par conséquent $A u \in C$.

$(\Leftarrow)$ Si $A u \in C$ alors $\forall \lambda \in K \backslash\{0\}, \exists v, \alpha$ tels que $A u=(A-\lambda I+\lambda I) u=$ $(A-\lambda I) v+\alpha f$. Donc $u=(A-\lambda I)\left(\lambda^{-1}(v-u)\right)+\alpha \lambda^{-1} f$ et par conséquent $u \in C_{1}=C$.

\section{Corollaire 2.4.}

$$
\forall \lambda \in K, \quad(A-\lambda I) u \in C \Leftrightarrow u \in C .
$$

Démonstration. Evidente.

Soit $P^{\prime}$ la projection orthogonale sur $C$ et $a \notin K$ (de sorte que $\forall \lambda \in K$, $a-\lambda \neq 0)$. Posons: $D\left(A^{\prime}\right)=D(A)+C ; \forall u \in D\left(A^{\prime}\right), u=v+w \quad(v \in D(A)$, $w \in C) \quad A^{\prime} u=\left(I-P^{\prime}\right) A v+a P^{\prime} u$.

Proposition 2.5. (i) $A^{\prime}$ est bien défini, linéaire et fermé,

(ii) $\mathrm{Co}\left(A^{\prime}\right)=C$ et $K \subseteq \operatorname{reg}\left(A^{\prime}\right)$.

Démonstration. (i) Soit $u \in D\left(A^{\prime}\right)$, avec $u=v_{1}+w_{1}=v_{2}+w_{2}$. Alors

$$
\left(I-P^{\prime}\right) A v_{1}-\left(I-P^{\prime}\right) A v_{2}=\left(I-P^{\prime}\right) A\left(v_{1}-v_{2}\right) \text {. }
$$

Or $v_{1}-v_{2}=w_{2}-w_{1} \in D(A) \cap C$ et par conséquent le Lemme 2.3 entraîne que $\left(I-P^{\prime}\right) A\left(v_{1}-v_{2}\right)=0$. Donc $A^{\prime}$ est bien défini. La linéarité de $A^{\prime}$ est une conséquence immédiate de celle de $A$.

(ii) Montrons maintenant que

$$
\forall \lambda \in K, \quad R\left(A^{\prime}-\lambda I\right)=R(A-\lambda I) \oplus\{f\} .
$$

Remarquons d'abord que si $u \in D\left(A^{\prime}\right)$, avec $u=v+w$ alors: $\left(A^{\prime}-\lambda I\right) u=$ $\left(I-P^{\prime}\right)(A-\lambda I) v+(a-\lambda) P^{\prime} u$ car $\left(I-P^{\prime}\right) v=\left(I-P^{\prime}\right) u$. Donc $\left(A^{\prime}-\lambda I\right) u \in$ $R(A-\lambda I)+C \subseteq R(A-\lambda I) \oplus\{f\}$. Inversement, soit $v \in D(A)$; posons

$$
u=(a-\lambda)^{-1} P^{\prime}(A-\lambda I) v+\left(I-P^{\prime}\right) v=v+(a-\lambda)^{-1} P^{\prime}(A-\lambda I) v-P^{\prime} v .
$$

Alors $u \in D\left(A^{\prime}\right)$ et $\left(A^{\prime}-\lambda I\right) u=\left(I-P^{\prime}\right)(A-\lambda I) v+P^{\prime}(A-\lambda I) v=(A-\lambda I) v$. Par conséquent $R(A-\lambda I) \subseteq R\left(A^{\prime}-\lambda I\right)$. Enfin $f=(a-\lambda)^{-1}\left(A^{\prime}-\lambda I\right) f \in R\left(A^{\prime}-\lambda I\right)$ d'où $\{f\} \subseteq R\left(A^{\prime}-\lambda I\right)$. Donc: $R(A-\lambda I) \oplus\{f\} \subseteq R\left(A^{\prime}-\lambda I\right)$ et (1) est démontré. On en déduit en particulier que $\forall \lambda \in K, R\left(A^{\prime}-\lambda I\right)$ est fermé. Montrons maintenant que

$$
\forall \lambda \in K, \quad N\left(A^{\prime}-\lambda I\right)=\{0\}
$$


En effet, soit $u \in N\left(A^{\prime}-\lambda I\right), u=v+w$. Alors $\left(I-P^{\prime}\right)(A-\lambda I) v+(a-\lambda) P^{\prime} u=0$ d'où $\left(I-P^{\prime}\right)(A-\lambda I) v=0$ et $P^{\prime} u=0$. Donc $(A-\lambda I) v \in C$, d'après le Corollaire 2.4, $v \in C$ et $P^{\prime} v=v$. Par conséquent $0=P^{\prime} u=P^{\prime} v+P^{\prime} w=$ $v+w=u$, ce qui établit (2).

(1) et (2) entraînent d'une part que $K \subseteq \operatorname{reg}\left(A^{\prime}\right)$, et d'autre part que $A^{\prime}$ est fermé (cf. [7, Proposition 2.2.3]). Donc (i) est démontré.

En outre (1) et $K \subseteq \operatorname{reg}\left(A^{\prime}\right)$ impliquent que $\operatorname{Co}\left(A^{\prime}\right)=C$ et (ii) est démontré.

Remarque. $\operatorname{codim}\left(R\left(A^{\prime}\right)\right)=\operatorname{codim}(R(A))-1$.

Proposition 2.6. Sous les hypothèses du Théorème 2.1, il existe $F$ et $E$ deux sous-espaces fermés de $H$ tels que $\forall \lambda \in K$,

$$
R(A-\lambda I) \oplus F=H \quad \text { et } \quad N(A-\lambda I) \oplus E=H .
$$

Démonstration. Posons $n=\operatorname{codim}(R(A))$.

Si $n=1$ alors on prend $F=\left\{f_{A}\right\}$, où $f_{A}$ est le vecteur introduit dans le Lemme 2.2.

Si $n>1$ on définit inductivement une suite $\left\{A_{n}\right\}$ d'opérateurs et une suite $\left\{u_{n}\right\}$ de vecteurs de la manière suivante (les notations sont celles du Lemme 2.2 et de la Proposition 2.4):

$$
A_{0}=A, \quad A_{j+1}=\left(A_{j}\right)^{\prime} \quad \text { et } \quad u_{j+1}=f_{A_{j}}, \quad j=0,1, \ldots, n-1 .
$$

On prends alors $F=\operatorname{clm}\left\{u_{1}, u_{2}, \ldots, u_{n}\right\}$. D'après la Remarque précédente, $\operatorname{codim}(R(A-\lambda I)+F)=n-n=0$, donc $R(A-\lambda I)+F=H$ et comme $R(A-\lambda I) \cap F=\{0\}$ alors $R(A-\lambda I) \oplus F=H$. De même, d'après [7, Proposition 3.35] on voit que $\rho_{\phi}^{r}\left(A^{*}\right)=\left\{\lambda ; \bar{\lambda} \in \rho_{\phi}^{r}(A)\right\}$. On procède donc comme ci-dessus à partir de $A^{*}$ et on obtient $G^{*}$ tel que $R\left(A^{*}-\overline{\lambda I}\right) \oplus G^{*}=H$. On prend alors $E=\left(G^{*}\right)^{\perp}$ et la proposition est démontrée.

Démonstration du Théorème 2.1. Conséquense directe des Propositions (2.6) et (1.4).

Soit $\varepsilon>0$ et soit $\mathscr{U}$ une composante connexe de $\rho_{\phi}(A)$. Posons:

$$
C_{\varepsilon}=\left\{\lambda \in \rho_{\phi}(A) ; \operatorname{dist}\left(\lambda, \sigma_{\phi}(A)\right) \geq \varepsilon \text { et }|\lambda| \leq 1 / \varepsilon\right\}
$$

où $\sigma_{\phi}(A)=\mathbb{C} \backslash \rho_{\phi}(A)$, spectre essentiel de $A$.

Remarque. $\forall \varepsilon>0, C_{\varepsilon}$ est compact et $\forall K$, compact contenu dans $\mathscr{U}, \exists \varepsilon>0$ tel que $K \subseteq C_{\varepsilon}$.

Dorénavant, pour tout opérateur $A, B$, etc., et tout sous-espace $M, N$, etc. ... de $H$ notons $A_{M}, B_{N}$, etc. ... respectivement la restriction de $A$ à $M$, de $B$ à $N$, etc. ...

Proposition 2.7. $\forall \varepsilon>0$ il existe un résolvant généralisé de $A$ vérifiant l'identité de la résolvante et méromorphe dans $\mathscr{U}$ sauf éventuellement sur un ensemble dénombrable $X$ de points situés à une distance inférieure à $\varepsilon$ de la frontière de $\mathscr{U}$ ou de norme $\geq 1 / \varepsilon$ (si $\mathscr{U}$ est bornée cette dernière condition peut être éliminée). En outre les projections $P_{\lambda}$ et $Q_{\lambda}$ associées au résolvant généralisé sont continues partout dans $\mathscr{U}$ sauf éventuellement sur $X$.

Démonstration. Posons $K=C_{\varepsilon}$. Alors $K \cap[\mathcal{U} \backslash \operatorname{reg}(A)]$ est un ensemble fini (puisque les $\lambda$ tels que $A-\lambda I$ est de Fredholm et n'est pas régulier sont des points isolés). Notons $\left\{\lambda_{1}, \lambda_{2}, \ldots, \lambda_{n}\right\}$ cet ensemble. Alors (cf. Proposition 
5.1.2 de [7]) $H$ est la somme directe finie de $n+1$ sous-espaces invariants $N_{0}, N_{1}, \ldots, N_{n}$ tels que: $(A-\lambda I)_{N_{0}}$ est régulier $\forall \lambda \in K ;\left(A-\lambda_{j}\right) I_{N_{j}}$ est nilpotent pour $j=1,2, \ldots, n$ et notons $T_{j}(0 \leq j \leq n)$ les projections de $H$ sur $N_{j}$ correspondant à cette décomposition.

Enfin soit $\operatorname{Rg}\left(A_{0}, \lambda\right)$ le résolvant généralisé associé à $(A-\lambda I)_{N_{0}}$, analytique dans $\mathscr{U}$ sauf éventuellement sur un ensemble dénombrable $X$, dont l'existence découle du Théorème 2.1. Alors $d$ étant le degré de la décomposition de Kato associée à $A-\lambda_{j} I$ on pose:

$$
\begin{gathered}
R_{j}(A, \lambda)=-\frac{1}{\lambda-\lambda_{j}} \sum_{i=0}^{d-1}\left(\frac{A-\lambda_{j}}{\lambda-\lambda_{j}}\right)^{i}, \\
\operatorname{Rg}(A, \lambda)=\operatorname{Rg}\left(A_{0}, \lambda\right) T_{0}+\sum_{j=1}^{n} R_{j}(A, \lambda) T_{j}
\end{gathered}
$$

et on vérifie sans peine que $\operatorname{Rg}(A, \lambda)$ répond à toutes les conditions du théorème et que les projections $P_{\lambda}$ et $Q_{\lambda}$ qui lui sont associées sont continues sur $\mathscr{U} \backslash X$.

Corollaire 2.8. Soit $A$ an opérateur fermé avec $D(A)$ dense dans $H$ et $\varepsilon>0$. Alors il existe un résolvant généralisé de $A$ vérifiant l'identité de la résolvante et analytique dans $\rho_{\phi}^{r}(A)$, méromorphe dans $\rho_{\phi}(A)$, sauf éventuellement sur un ensemble dénombrable de points situés à une distance inférieure à $\varepsilon$ de la frontière de $\rho_{\phi}(A)$ ou de norme $\geq 1 / \varepsilon$.

Démonstration. C'est une conséquence immédiate de la proposition précédente: il suffit de construire le résolvant généralisé séparément sur chaque composante connexe.

Remarque. Ce dernier corollaire est une généralisation de [5, Theorem 1] au cas des opérateurs fermés à domaine dense.

\section{COMPLÉMENTS SUR LES OPÉRATEURS QUASI-FREDHOLM}

Les opérateurs quasi-Freholm ont été introduits par dans [7], comme une généralisation des opérateurs semi-Fredholm. Dans ce travail on n'aura besoin que d'une de leurs caractérisations: Si A est fermé avec un domaine dense alors il est quasi-Fredholm de degré $d$ et on note $A \in q \Phi(d)$ si et seulement si il existe $M$ et $N$ deux sous-espaces fermés de $H$ invariants par $A$, tels que:

(a) $H=M \oplus N$,

(b) $N \subseteq D(A)$ et $A_{N}$ est nilpotent de degré $d$,

(c) $0 \in \operatorname{reg}\left(A_{M}\right)$.

Le couple $(M, N)$ est une décomposition de Kato de degré $d$ associée à $A$. Notons que $d=\inf \left\{n \in \mathbb{N} ; \forall m \geq n: R\left(A^{m}\right) \cap N(A)=R\left(A^{n}\right) \cap N(A)\right\}$.

Remarque. A est régulier $\Leftrightarrow A \in q \Phi(0)$. Dans la suite on notera $\rho_{q \phi}(A)=$ $\{\lambda \in \mathbb{C} ; \exists d \in \mathbb{N}$ tel que $A-\lambda I \in q \Phi(d)\}$, et $\rho_{q \phi}^{s}(A)=\{\lambda \in \mathbb{C} ; A-\lambda I \in q \Phi(d)$ avec $d \geq 1\}$, alors: $\rho_{q \phi}(A)=\operatorname{reg}(A) \cup \rho_{q \phi}^{s}(A)$.

Avec les notations de [3 et 4] on trouve:

$$
\rho_{\mathrm{S}-\mathrm{F}}(A) \subseteq \rho_{q \phi}(A), \quad \rho_{\mathrm{S}-\mathrm{F}}(A) \subseteq \rho_{q \phi}^{s}(A) \quad \text { et } \quad \rho_{\mathrm{S}-\mathrm{F}}^{r}(A) \subseteq \operatorname{reg}(A) .
$$

Proposition 3.1. (i) $\rho_{q \phi}^{s}(A)$ est dénombrable et n'admet pas de points d'accumulation dans $\rho_{q \phi}(A)$.

(ii) Si $A \in q \Phi(d)$ alors $\operatorname{Co}(A)=\bigcap_{j \geq 0} R\left(A^{j}\right)$ est fermé. 
Démonstration. (i) Se déduit de [7, Proposition 4.3.1(a)].

(ii) On utilise la décomposition de Kato et la Proposition 1.2.

Proposition 3.2. Soit $A$ an opérateur fermé. Alors les conditions suivantes sont équivalentes:

(i) $A$ est quasi-Fredholm,

(ii) $C(A)=\bigcap_{j \geq 0} R\left(A^{j}\right)$ et $H(A)=\bigcup_{j \geq 0} N\left(A^{j}\right)$ sont fermés; alors

$$
\lim _{\lambda \rightarrow 0} P_{N(A-\lambda I)}=P_{N(A) \cap C(A)} ; \quad \lim _{\lambda \rightarrow 0} P_{R(A-\lambda I)}=P_{R(A)+H(A)} .
$$

Démonstration. (i) $\Rightarrow$ (ii). Soit $A$ quasi-Fredholm et $M, N$ une décomposition de Kato de degré $d$ associée à. A. Il est facile de voir que si $\lambda \neq 0$ alors $N(A-\lambda I) \subseteq C(A)$ (dans ce cas $C(A)=\operatorname{Co}(A)$ ). Or, $\operatorname{Co}(A) \subseteq M$ d'où $N(A-\lambda I)=N\left((A-\lambda I) \cap M=N\left((A-\lambda I)_{M}\right)\right.$. Donc $P_{N(A-\lambda I)}=P_{N\left((A-\lambda I)_{M}\right)}$ et comme $0 \in \operatorname{reg}\left(A_{M}\right) \Rightarrow \lambda \mapsto P_{N\left((A-\lambda I)_{M}\right)}$ est continue en zéro, on déduit que

$$
\lim _{\lambda \rightarrow 0} P_{N(A-\lambda I)}=\lim _{\lambda \rightarrow 0} P_{N\left((A-\lambda I)_{M}\right)}=P_{N\left(A_{M}\right)}=P_{N(A) \cap \operatorname{Co}(A)} .
$$

Montrons maintenant la deuxième condition de (2). Pour cela remarquons que

$$
R(A)+\bigcup_{j \geq 0} N\left(A^{j}\right)=A(M \cap D(A)) \oplus N .
$$

En effet, l'inclusion “ $\supseteq$ ” est évidente; montrons l'autre inclusion.

$$
\begin{gathered}
N \subseteq D(A) \Rightarrow R(A)=A(M \cap D(A)) \oplus A(N) \subseteq A(M \cap D(A)) \oplus N, \\
N \subseteq \bigcup_{j \geq 0} N\left(A^{j}\right) \Rightarrow \bigcup_{j \geq 0} N\left(A^{j}\right)=\bigcup_{j \geq 0} N\left(A^{j}\right) \cap M \oplus N=\bigcup_{j \geq 0} N\left(\left(A_{M}\right)^{j}\right) \oplus N
\end{gathered}
$$

et comme $A_{M}$ est régulier $\Rightarrow \bigcup_{j \geq 0} N\left(\left(A_{M}\right)^{j}\right) \subseteq R\left(A_{M}\right)=A(M \cap D(A))$, on en déduit que

$$
\bigcup_{j \geq 0} N\left(A^{j}\right) \subseteq A(M \cap D(A)) \oplus N .
$$

Or (2) et $(3) \Rightarrow(1)$. Par ailleurs si $\lambda \neq 0$ alors $R(A-\lambda I)=R\left((A-\lambda) I_{M}\right) \oplus$ $N=(A-\lambda I)(M \cap D(A)) \oplus N$ donc $P_{R(A-\lambda I)}=P_{(A-\lambda I)(M \cap D(A)) \oplus N}$ et comme $0 \in \operatorname{reg}\left(A_{M}\right) \Rightarrow \lambda \mapsto P_{R\left((A-\lambda I)_{M}\right)}$ est continue on zéro, on déduit que

$$
\lim _{\lambda \rightarrow 0} P_{R(A-\lambda I)}=\lim _{\lambda \rightarrow 0} P_{R\left((A-\lambda I)_{M}\right) \oplus N}=P_{R\left(A_{M}\right) \oplus N}
$$

en utilisant [7, Corollaire 1.3.5] et (1) permet de conclure.

(ii) $\Rightarrow$ (i). Sous hypothèse (ii) on en déduit que $N(A) \cap \bigcap_{j \geq 0} R\left(A^{j}\right)$ et $R(A)+\bigcup_{j \geq 0} N\left(A^{j}\right)$ sont fermés dans $H$. La Proposition 3.3.6 de [7] montre alors que $\bar{A}$ est quasi-Fredholm.

Théorème 3.3. Soit $A$ un opérateur fermé avec $D(A)$ dense dans $H$ et tel que $A \in q \Phi(d)$ alors $\exists \varepsilon>0$ tel que $\forall \lambda \in \mathbb{C},|\lambda|<\varepsilon$ on $a$ :

(a) $\operatorname{Co}(A-\lambda I)+H_{0}(A-\lambda I)=\operatorname{Co}(A)+H_{0}(A)$.

(B) $\operatorname{Co}(A-\lambda I) \cap \overline{H_{0}(A-\lambda I)}=\operatorname{Co}(A) \cap \overline{H_{0}(A)}$. 
Démonstration. Soit $A \in q \Phi(d)$ et $M, N$ une décomposition de Kato de degré $d$ associée à $A$.

Montrons que

$$
H_{0}(A) \cap M=H_{0}(A) \cap \operatorname{Co}(A) .
$$

En effet, $\operatorname{Co}(A) \subseteq M$ et $H_{0}\left(A_{M}\right) \subseteq \operatorname{Co}(A)$ (car $A_{M}$ est régulier) impliquent que

$$
\begin{aligned}
H_{0}(A) \cap \operatorname{Co}(A) & =H_{0}(A) \cap M \cap \operatorname{Co}(A)=H_{0}\left(A_{M}\right) \cap \operatorname{Co}(A) \\
& =H_{0}\left(A_{M}\right)=H_{0}(A) \cap M .
\end{aligned}
$$

Montrons que

$$
\mathrm{Co}(A)+H_{0}(A)=\mathrm{Co}(A) \oplus N .
$$

En effet, $N \subseteq N\left(T^{d}\right) \subseteq H_{0}(A)$ et (1) impliquent d'une part $\operatorname{Co}(A)+N \subseteq$ $\mathrm{Co}(A)+H_{0}(A)$ et d'autre part

$$
H_{0}(A)=H_{0}(A) \cap M \oplus N=H_{0}(A) \cap \operatorname{Co}(A) \oplus N \subseteq \operatorname{Co}(A) \oplus N
$$

d'où $\mathrm{Co}(A)+H_{0}(A) \subseteq \operatorname{Co}(A) \oplus N$ et par conséquent (2) est démontré.

$$
A_{N} \text { nilpotent implique que } \forall \lambda \neq 0 \quad(A-\lambda I)_{N} \text { est inversible }
$$

$\operatorname{reg}\left(A_{M}\right)$ est ouvert donc $\exists \varepsilon>0$ tel que $\forall \lambda \in \mathrm{C}$,

$$
|\lambda|<\varepsilon \Rightarrow 0 \in \operatorname{reg}\left((A-\lambda I)_{M}\right) ;
$$

donc d'après le Théorème 1.1 on a:

$$
\operatorname{Co}\left((A-\lambda I)_{M}\right)=\operatorname{Co}\left(A_{M}\right)=\operatorname{Co}(A) .
$$

On outre, $\forall \lambda \neq 0 \quad(A-\lambda I)_{N}$ est inversible, d'où $\forall \lambda \in \mathrm{C}, 0<|\lambda|<\varepsilon \Rightarrow 0 \in$ $\operatorname{reg}(A-\lambda I)$. Par conséquent, $H_{0}(A-\lambda I) \subseteq \operatorname{Co}(A-\lambda I)$ et (3) impliquent en particulier que $(A-\lambda I)(N)=N$. Donc $\forall \lambda \in \mathbb{C}, 0<|\lambda|<\varepsilon$ implique que

$$
\begin{aligned}
& H_{0}(A-\lambda I)+\operatorname{Co}(A-\lambda I)=\operatorname{Co}(A-\lambda I) \\
& \quad=\operatorname{Co}(A-\lambda I) \cap M+N=\operatorname{Co}(A)+N=\operatorname{Co}(A)+H_{0}(A) .
\end{aligned}
$$

D'où finalement $\forall \lambda \in \mathbb{C}, 0<|\lambda|<\varepsilon \Rightarrow H_{0}(A-\lambda I)+\operatorname{Co}(A-\lambda I)=\operatorname{Co}(A)$ $+H_{0}(A)$; d'où $(\alpha)$ est démontré. Pour démontrer $(\beta)$ on remarque que $A \in q \phi(d) \Leftrightarrow A^{*} \in q \phi(d)$ (cf. [7, Proposition 3.3.5]). Alors $\operatorname{Co}\left(A^{*}-\overline{\lambda I}\right)+$ $H_{0}\left(A^{*}-\overline{\lambda I}\right)=\mathrm{Co}\left(A^{*}\right)+H_{0}\left(A^{*}\right)$ et on déduit $(\beta)$ de la Proposition 1.2(ii) en y remplacạnt $A$ par $A^{*}$.

Corollaire 3.4. Soit $A$ un opérateur fermé avec $D(A)$ dense dans $H$. Alors les applications

$$
\lambda \mapsto \operatorname{Co}(A-\lambda I)+H_{0}(A-\lambda I), \quad \lambda \mapsto \operatorname{Co}(A-\lambda I) \cap \overline{H_{0}(A-\lambda I)}
$$

sont constantes dans chaque composante connexe du domaine quasi-Fredholm $\left(\rho_{q \phi}(A)\right)$ de $A$.

Remarque. L'application $\lambda \mapsto \mathrm{Co}(A-\lambda I)+\overline{H_{0}(A-\lambda I)}$ est constante dans chaque composante connexe du domaine quasi-Fredholm $\left(\rho_{q \phi}(A)\right)$ de $A$.

En effet dans la démonstration du Théorème 3.3 on a montré (cf. (2)) que $\mathrm{Co}(A)+H_{0}(A)=\operatorname{Co}(A) \oplus N$.

Or, $\mathrm{Co}(A) \oplus N$ est fermé dans $H$; en effet $H=M \oplus N \Rightarrow H$ est isomorphe à $M \times N$ donc $\operatorname{Co}(A) \oplus N$ est isomorphe à $\operatorname{Co}(A) \times N$ qui est fermé dans $M \times N$ 
et par conséquent, $\operatorname{Co}(A) \oplus N$ est fermé dans $H$ donc $\operatorname{Co}(A)+H_{0}(A)$ est fermé, par conséquent, $\operatorname{Co}(A-\lambda I)+H_{0}(A-\lambda I)$ est fermé dans chaque composantes connexe de $\rho_{q \phi}(A) ;$ donc, $\operatorname{Co}(A-\lambda I)+H_{0}(A-\lambda I)=\operatorname{Co}(A-\lambda I)+\overline{H_{0}(A-\lambda I)}$ pour tout $\lambda \in \rho_{q \phi}(A)$.

\section{TRIANGUlarisAtion D'APOSTOL GÉNÉRALISÉE}

Soit $B(H)$, l'espace des opérateurs bornés de $H$ dans lui-même, et $T \in$ $B(H)$. D'après la Proposition 1.2(i), $\mathrm{Co}(\lambda I-T)$ est constant dans chaque composante connexe de $\operatorname{reg}(T)$. Il est facile de voir que les composantes connexes de $\operatorname{reg}(T)$ constituent un ensemble dénombrable. Soit $S=\left\{\lambda_{i}\right\}$ l'ensemble obtenu en choisissant un et seul $\lambda_{i}$ dans chaque composante connexe.

Alors

$$
\bigcap_{\lambda \in \operatorname{reg}(T)} \operatorname{Co}(\lambda I-T)=\bigcap_{\lambda_{i} \in S} \operatorname{Co}\left(\lambda_{i} I-T\right) .
$$

Posons:

$$
\widetilde{H}_{r}(T)=\left[\bigcap_{\lambda_{i} \in S} \operatorname{Co}\left(\lambda_{i} I-T\right)^{*}\right]^{\perp}, \quad \widetilde{H}_{l}(T)=\left[\bigcap_{\lambda_{i} \in S} \operatorname{Co}\left(\lambda_{i} I-T\right)\right]^{\perp} .
$$

On notera dans la suite $\widetilde{H}_{r}=\widetilde{H}_{r}(T)$ et $\widetilde{H}_{l}=\widetilde{H}_{l}(T)$.

Lemme 4.1. Soit $T \in B(H)$ alors:

(a) $\widetilde{H}_{r}=\operatorname{clm}\{N(\lambda I-T)\}_{\lambda \in \operatorname{reg}(T)}$,

(b) $\widetilde{H}_{l}=\operatorname{clm}\left\{N(\lambda I-T)^{*}\right\}_{\lambda \in \operatorname{reg}(T)}$.

Démonstration. (a) On a $\forall \lambda \in \operatorname{reg}(T) \bigcap_{\lambda_{i} \in S} \operatorname{Co}\left(\lambda_{i} I-T\right)^{*} \subseteq R(\lambda I-T)^{*}$ et comme $\forall \lambda \in \mathbb{C}, N(\lambda I-T)=R(\lambda I-T)^{* \perp}$, on en déduit que

$$
\forall \lambda \in \operatorname{reg}(T) \quad N(\lambda I-T)=R(\lambda I-T)^{* \perp} \subseteq\left[\bigcap_{\lambda_{i} \in S} \operatorname{Co}\left(\lambda_{i} I-T\right)^{*}\right]^{\perp}=\widetilde{H}_{r}
$$

d'où $\operatorname{clm}\{N(\lambda I-T)\}_{\lambda \in \operatorname{reg}(T)} \subseteq \widetilde{H}_{r}$.

Réciproquement, soit $u \perp \operatorname{clm}\left\{N(\lambda I-T\}_{\lambda \in \operatorname{reg}(T)}\right.$, alors en particulier $\forall \lambda \in$ $\operatorname{reg}(T) u \perp N(\lambda I-T)$, donc $\forall \lambda \in \operatorname{reg}(T), u \in R(\lambda I-T)^{*}$ par conséquent $u \in \bigcap_{\lambda \in \operatorname{reg}(T)} R(\lambda I-T)^{*}=\widetilde{H}_{r}^{\perp}$ d'où

$$
\left[\operatorname{clm}\{N(\lambda I-T)\}_{\lambda \in \operatorname{reg}(T)}\right]^{\perp} \subseteq \widetilde{H}_{r}^{\perp} \Rightarrow \widetilde{H}_{r} \subseteq \operatorname{clm}\{N(\lambda I-T)\}_{\lambda \in \operatorname{reg}(T)}
$$

donc (a) est démontré.

(b) Se démontre de la même façon que (a).

Lemme 4.2. $\lambda \in \operatorname{reg}(T)$ alors $N(\lambda I-T)^{*} \subseteq\left[\operatorname{clm}\{N(\mu I-T)\}_{\mu \in \mathbb{C}}\right]^{\perp}$.

Démonstration. On a $\forall \mu \neq \lambda N(\mu I-T) \subseteq R(\lambda I-T)$, d'autre part si $\lambda \in \operatorname{reg}(T)$ alors $N(\lambda I-T) \subseteq R(\lambda I-T)$. Par conséquent $\forall \mu \in \mathbb{C}, N(\mu I-T) \subseteq R(\lambda I-T)$ et comme $R(\lambda I-T)$ est fermé on en déduit que $\operatorname{clm}\{N(\mu I-T)\}_{\mu \in \mathbb{C}} \subseteq R(\lambda I-T)$, d'où

$$
N(\lambda I-T)^{*}=R(\lambda I-T)^{\perp} \subseteq\left[\operatorname{clm}\{N(\mu I-T)\}_{\mu \in \mathbb{C}}\right]^{\perp} .
$$

Lemma 4.3. $\widetilde{H}_{r} \perp \widetilde{H}_{l}$.

Démonstration. Les Lemmes 4.1 et 4.2 impliquent que $\forall \lambda \in \operatorname{reg}(T) N(\lambda I-T)^{*} \subseteq$ $\widetilde{H}_{r}^{\perp}$ et donc $\widetilde{H}_{l} \subseteq \widetilde{H}_{r}^{\perp}$. 
Remarque. $\widetilde{H}_{r} \oplus \widetilde{H}_{l}$ est fermé car c'est une somme orthogonale de sous-espaces fermés (Lemme 4.3).

Soit $\widetilde{H}_{0}=\left[\widetilde{H}_{r} \oplus \widetilde{H}_{l}\right]^{\perp}$. Alors $H=\widetilde{H}_{r} \oplus \widetilde{H}_{0} \oplus \widetilde{H}_{l}$.

$\widetilde{H}_{r}$ est invariant par $T$, on notera $T_{r}$ la restriction de $T$ à $\widetilde{H}_{r}$. En général $\widetilde{H}_{0}$ et $\widetilde{H}_{l}$ ne sont pas invariants par $T$, on définit $T_{0}$ et $T_{l}$ comme des compressions c'est à dire $T_{0} \in B\left(\widetilde{H}_{0}\right)$, si $u_{0} \in \widetilde{H}_{0}$ alors $T_{0} u_{0}=P_{0} T u_{0}$ où $P_{0}$ est la projection orthogonale sur $\widetilde{H}_{0} . T_{l}$ est défini de la même fận que $T_{0}$.

On remarque que si $u_{0} \in \widetilde{H}_{0}$ et $u_{l} \in \widetilde{H}_{l}$ alors $\left(T u_{0}, u_{l}\right)=\left(u_{0}, T^{*} u_{l}\right)=0$ (car $\widetilde{H}_{l}$ est invariant par $T^{*}$ ), d'où $T\left(\widetilde{H}_{0}\right)$ est orthogonal à $\widetilde{H}_{l}$.

On peut représenter $T$ par une matrice $3 \times 3$. Si $u=u_{r}+u_{0}+u_{l}$ alors

$$
T u=T u_{r}+T_{0} u_{0}+\left(I-P_{0}\right) T u_{0}+T_{l} u_{l}+\left(I-P_{l}\right) T u_{l}
$$

dans cette expression on a: $T u_{r} \in \widetilde{H}_{r}, T_{0} u_{0} \in \widetilde{H}_{0},\left(I-P_{0}\right) T u_{0} \in \widetilde{H}_{r}, T_{l} u_{l} \in \widetilde{H}_{l}$ et $\left(I-P_{l}\right) T u_{l} \in \widetilde{H}_{r} \oplus \widetilde{H}_{0}$.

$$
T=\left(\begin{array}{ccc}
T_{r} & A & B \\
0 & T_{0} & C \\
0 & 0 & T_{l}
\end{array}\right) .
$$

où

$$
\begin{array}{ll}
A u_{0}=\left(I-P_{0}\right) T u_{0} & \text { donc } A \in B\left(\widetilde{H}_{0}, \widetilde{H}_{r}\right), \\
B u_{l}=P_{r}\left(I-P_{l}\right) T u_{l}=P_{r} T u_{l} & \text { donc } B \in B\left(\widetilde{H}_{l}, \widetilde{H}_{0}\right), \\
C u_{l}=P_{0}\left(I-P_{l}\right) T u_{l}=P_{0} T u_{l} & \text { donc } C \in B\left(\widetilde{H}_{l}, \widetilde{H}_{0}\right) .
\end{array}
$$

Remarque 4.4. $\left(T_{r}\right)^{*}=T_{l}^{*} ;\left(T_{l}\right)^{*}=T_{r}^{*} ;\left(T_{0}\right)^{*}=T_{0}^{*}$.

Montrons par exemple la dernière égalité. Soit $u \in \widetilde{H}_{0}, \forall v \in \widetilde{H}_{0}$ on a

$$
\left(T_{0}^{*} u, v\right)=\left(P_{0} T^{*} u, v\right)=\left(T^{*} u, v\right)=(u, T v)=\left(u, P_{0} T v\right)+\left(u,\left(I-P_{0}\right) T v\right)
$$

or, $\left(I-P_{0}\right) T v \in \widetilde{H}_{r} \oplus \widetilde{H}_{l}=\widetilde{H}_{0}^{\perp}$ donc $\left(u,\left(I-P_{0}\right) T v\right)=0$ et par conséquent

$$
\left(T_{0}^{*} u, v\right)=\left(u, P_{0} T v\right)=\left(\left(P_{0} T_{\widetilde{H}_{0}}\right)^{*} u, v\right) \quad \forall v \in \widetilde{H}_{0}
$$

d'où $T_{0}^{*}=\left(T_{0}\right)^{*}$.

Proposition 4.5. Soit $T \in B(H)$ alors:

$$
\operatorname{reg}(T)=\left\{\mu \in \rho_{q \phi}(T) ; N(\mu I-T) \subseteq \operatorname{clm}\{N(\lambda I-T)\}_{\lambda \neq \mu}\right\} .
$$

Démonstration. Montrons l'inclusion suivante: $\left\{\mu \in \rho_{q \phi}(T) ; N(\mu I-T) \subseteq\right.$ $\left.\operatorname{clm}\{N(\lambda I-T)\}_{\lambda \neq \mu}\right\} \subseteq \operatorname{reg}(T)$. Remarquons que $\forall \lambda \neq \mu$ on a $N(\lambda I-T) \subseteq$ $\operatorname{Co}(\mu I-T)$, d'autre part $\mu \in \rho_{q \phi}(T) \Rightarrow \operatorname{Co}(\mu I-T)$ est fermé d'où $N(\mu I-T) \subseteq$ $\operatorname{clm}\{N(\lambda I-T)\}_{\lambda \neq \mu} \subseteq \operatorname{Co}(\mu I-T)$ et par conséquent $\mu I-T$ est quasi-Fredholm de degré zéro ou encore $\mu \in \operatorname{reg}(T)$.

L'inclusion inverse se déduit du Théorème 1.1(iv).

Corollaire 4.6. $T \in B(H)$ alors $\rho_{q \phi}^{s}(T) \subseteq \sigma_{p}(T)$.

Démonstration. $\mu \in \rho_{q \phi}^{s}(T)$, par la proposition précédente on a $N(\mu I-T) \not \subset$ $\operatorname{clm}\{N(\lambda I-T)\}_{\lambda \neq \mu}$ ce qui entraine que $N(\mu I-T) \neq\{0\}$.

Remarque 4.7. Si $\mu \in \operatorname{reg}(T)$ et $G$ est un voisinage de $\mu$ alors

$$
\operatorname{clm}\{N(\lambda I-T)\}_{\lambda \in G \backslash \mu}=\operatorname{clm}\{N(\lambda I-T)\}_{\lambda \in G} .
$$

Ce résultat est une conséquense directe de la Proposition 4.5. 
Lemme 4.8 (Kato [6, Lemme 3.3.1]). Soit $T \in B(H)$ avec $R(T)$ fermé et $Y$ un sous-espace fermé de $H$ tel que $Y+N(T)$ soit fermé. Alors $R\left(T_{Y}\right)$ est fermé.

On notera dans la suite

$$
\begin{aligned}
& \rho_{r}(T)=\{\lambda \in \mathbb{C} ;(\lambda I-T) \text { est surjective }\}, \\
& \rho_{l}(T)=\{\lambda \in \mathbb{C} ;(\lambda I-T) \text { est injective à image fermé }\} .
\end{aligned}
$$

Lemme 4.9. Soit $\mu \in \rho_{q \phi}(T)$ et $G$ un voisinage de $\mu$. Si on pose $Y=$ $\operatorname{clm}\{N(\lambda I-T)\}_{\lambda \in G \backslash \mu}$ alors $\mu \in \rho_{r}\left(T_{Y}\right)$.

Démonstration. Soit $\mu \in \rho_{q \phi}(T)$ et $(M, N)$ une décomposition de Kato associée à $\mu I-T$. On a d'une part $\forall \lambda \neq \mu, N(\lambda I-T) \subseteq \operatorname{Co}(\mu I-T) \subseteq M$ par conséquent $Y=\operatorname{clm}\left\{N\left((\lambda I-T)_{M}\right)\right\}_{\lambda \in G \backslash \mu}$. D'autre part, $N(\mu I-T)=$ $N\left((\mu I-T)_{M}\right) \oplus N\left((\mu I-T)_{N}\right)$ avec $\mu \in \operatorname{reg}\left(T_{M}\right)$, d'aprés la Remarque 4.7 on a $Y=\operatorname{clm}\left\{N\left((\lambda I-T)_{M}\right)\right\}_{\lambda \in G \backslash \mu}=\operatorname{clm}\left\{N\left((\lambda I-T)_{M}\right)\right\}_{\lambda \in G}$. En outre, $\mu \in \operatorname{reg}(T) \Rightarrow R\left((\mu I-T)_{M}\right)$ est fermé et comme $N\left((\mu I-T)_{M}\right) \subseteq Y$ en utilisant le Lemme 4.8, on en déduit que $R\left((\mu I-T)_{Y}\right)$ est fermé. Par ailleurs on a $\forall \lambda \neq \mu, N(\lambda I-T) \subseteq R\left((\mu I-T)_{Y}\right)$, d'où $Y \subseteq R\left((\mu I-T)_{Y}\right)$ ou encore $\mu \in \rho_{r}\left(T_{Y}\right)$.

Théorème 4.10. Soit $T \in B(H)$ alors on $a$ :

(i) $\rho_{q \phi}(T) \subseteq \rho_{r}\left(T_{r}\right) \cap \rho_{l}\left(T_{l}\right)$,

(ii) $\operatorname{reg}(T) \subseteq \rho\left(T_{0}\right)$,

(iii) $\operatorname{reg}(T)=\rho_{r}\left(T_{r}\right) \cap \rho_{l}\left(T_{l}\right) \cap \rho\left(T_{0}\right)$,

(iv) $\rho_{q \phi}^{s}(T) \subseteq \sigma_{p}^{0}\left(T_{0}\right)=\left\{\lambda \in \mathbb{C} ; \lambda\right.$ est une valeur propre isolée de $\left.T_{0}\right\}$.

Démonstration. (i) en utilisant le Lemme 4.9 et la Remarque 4.4, on obtient:

$$
\rho_{q \phi}(T) \subseteq \rho_{r}\left(T_{r}\right) \cap\left(\rho_{r}\left(T_{r}^{*}\right)\right)^{*}=\rho_{r}\left(T_{r}\right) \cap \rho_{l}\left(\left(T_{r}^{*}\right)^{*}\right)=\rho_{r}\left(T_{r}\right) \cap \rho_{l}\left(T_{l}\right) .
$$

(ii) D'après le Lemme 4.1, $\forall \mu \in \operatorname{reg}(T), N(\mu I-T) \subseteq \widetilde{H}_{r}$ et $N(\mu I-T)^{*} \subseteq$ $\widetilde{H}_{l}$. D'autre part $\widetilde{H}_{0}=\left[\widetilde{H}_{r}+\widetilde{H}_{l}\right]^{\perp}=\widetilde{H}_{r}^{\perp} \cap \widetilde{H}_{l}^{\perp}$. Donc $\forall \mu \in \operatorname{reg}(T), \mu I-T_{0}$ est injective à image dense dans $\widetilde{H}_{0}$. Il reste à montrer que $R\left(\mu I-T_{0}\right)$ est fermé. Pour cela remarquons que $(\mu I-T)\left(\widetilde{H}_{0}\right)$ est fermé; en effet, $R(\mu I-T)$ est fermé et $\widetilde{H}_{0} \oplus N(\mu I-T)$ l'est aussi (comme somme orthogonale de deux fermés). Donc $(\mu I-T)\left(\widetilde{H}_{0}\right)$ est fermé (cf. le Lemme 4.8). Par ailleurs,

$$
(\mu I-T)\left(\widetilde{H}_{0}\right)=P_{0}(\mu I-T)\left(\widetilde{H}_{0}\right) \oplus\left(I-P_{0}\right)(\mu I-T)\left(\widetilde{H}_{0}\right)
$$

d'où on déduit que $P_{0}(\mu I-T)\left(\widetilde{H}_{0}\right)$ est fermé ou encore que $R\left(\mu I-T_{0}\right)$ est fermé. Par conséquent $\operatorname{reg}(T) \subseteq \rho\left(T_{0}\right)$.

(iii) $\operatorname{reg}(T) \subseteq \rho_{r}\left(T_{r}\right) \cap \rho_{l}\left(T_{l}\right) \cap \rho\left(T_{0}\right)$ se déduit de (i) et (ii). Pour l'inclusion inverse, remarquons que $\forall \lambda \in \rho_{r}\left(T_{r}\right) \cap \rho_{l}\left(T_{l}\right) \cap \rho\left(T_{0}\right), N(\lambda I-T)=N\left(\lambda I_{r}-T_{r}\right)$ et comme $\lambda I_{r}-T_{r}$ est surjectif, on en déduit que $N(\lambda I-T) \subseteq \operatorname{Co}(\lambda I-T)$. Comme $c(\lambda I-T)=\min \left\{c\left(\lambda I_{r}-T_{r}\right), c\left(\lambda I_{l}-T_{l}\right), c\left(\lambda I_{0}-T_{0}\right)\right\}>0$ on voit que $R(\lambda I-T)$ est fermé $\forall \lambda \in \rho_{r}\left(T_{r}\right) \cap \rho_{l}\left(T_{l}\right) \cap \rho\left(T_{0}\right)$.

(iv) Soit $\mu \in \rho_{q \phi}^{s}(T)$. Supposons que $\mu$ n'est pas une valeur propre de $T_{0}$. Alors d'après la décomposition $H=\widetilde{H}_{r} \oplus \widetilde{H}_{0} \oplus \widetilde{H}_{l}$ et (i) on voit que $N\left(\mu I_{r}-T\right)=N\left(\mu I-T_{r}\right) \subseteq \widetilde{H}_{r}=(\mu I-T)\left(\widetilde{H}_{r}\right)$ d'où $N(\mu I-T) \subseteq \operatorname{Co}(\mu I-T)$ ce qui implique que $\mu \in \operatorname{reg}(T)$ ce qui est contraire à l'hypothèse $\mu \in \rho_{q \phi}^{s}(T)$. 
Donc $\mu \in \sigma_{p}\left(T_{0}\right)$ et la Proposition 3.1(i) entraîne que $\mu$ est isolé. Alors de (ii) on déduit que $\mu \in \sigma_{p}^{0}\left(T_{0}\right)$.

\section{SÉPARATION D'ENSEMBLeS FINIS DE POINTS SINGULIERS QUASI-FREDHOLM}

G. R. Allan $[1,2]$ a montré l'existence d'un inverse à droite (resp. à gauche) de $\lambda I-T$ analytique dans $\rho_{r}(T)$ (resp. $\rho_{l}(T)$ ). Les résultats de G. R. Allan et le Théorème 4.10(i) montrent qu'on peut trouver un inverse à droite de $\lambda I_{r}-T_{r}$, noté $R(\lambda)$, analytique dans $\rho_{q \phi}(T)$, et un inverse à gauche $L(\lambda)$ de $\lambda I_{l}-T_{l}$, analytique dans $\rho_{q \phi}(T)$.

On définit la fonction $F: \operatorname{reg}(T) \mapsto B(H)$, par la représentation matricielle relativement à la décomposition $H=\widetilde{H}_{r} \oplus \widetilde{H}_{0} \oplus \widetilde{H}_{l}$,

$$
F(\lambda)=\left(\begin{array}{lll}
R(\lambda) & R(\lambda) A R\left(\lambda, T_{0}\right) & R(\lambda)\left[A R\left(\lambda, T_{0}\right) C+B\right] L(\lambda) \\
0 & R\left(\lambda, T_{0}\right) & R\left(\lambda, T_{0}\right) C L(\lambda) \\
0 & 0 & L(\lambda)
\end{array}\right)
$$

où $R\left(\lambda, T_{0}\right)=\left(\lambda I_{0}-T_{0}\right)^{-1}$. Les résultats de [1,2] et le Théorème 4.10 montrent que la fonction $F(\lambda)$ est analytique dans $\operatorname{reg}(T)$.

Soit $\sigma$ une partie compacte de $\rho_{q \phi}^{s}(T)$ et $\Gamma$ une courbe de Jordan fermée rectifiable dans $\operatorname{reg}(T)$ et entourant $\sigma$. On définit la projection spectrale généralisée associée à $\sigma$ par:

$$
P(\sigma)=(2 i \pi)^{-1} \int_{\Gamma} F(\lambda) d \lambda
$$

dont la représentation matricielle est la suivante:

$$
P(\sigma)=\left(\begin{array}{ccc}
0 & (2 i \pi)^{-1} \int_{\Gamma} R(\lambda) A R\left(\lambda, T_{0}\right) d \lambda & (2 i \pi)^{-1} \int_{\Gamma} R(\lambda) A R\left(\lambda, T_{0}\right) C L(\lambda) d \lambda \\
0 & P_{0}(\sigma) & (2 i \pi)^{-1} \int_{\Gamma} R\left(\lambda, T_{0}\right) C L(\lambda) d \lambda \\
0 & 0 & 0
\end{array}\right)
$$

où $P_{0}(\sigma)=(2 i \pi)^{-1} \int_{\Gamma} R\left(\lambda, T_{0}\right) d \lambda$.

$F(\lambda)$ et $P(\sigma)$ ont les propriétés suivantes (cf. [4] et le Théorème 4.10).

(a) $F(\lambda)$ est un résolvent généralisé de $T$ analytique dans $\operatorname{reg}(T)$ (ne vérifiant pas en général l'identité de la résolvante),

(b) $P^{2}(\sigma)=P(\sigma)$.

(c) $P(\sigma) T=T P(\sigma)$.

(d) Soit $T_{\sigma}$ la restriction de $T$ au sous-espace invariant $P(\sigma)(H)$; alors $\sigma\left(T_{\sigma}\right)=\sigma$.

(e) Soit $P^{\prime}(\sigma)=I-P(\sigma)$ et $T_{\sigma}^{\prime}$ la restriction de $T$ au sous-espace invariant $P^{\prime}(\sigma)(H)$ alors

$$
\operatorname{reg}(T) \cup \sigma \subseteq \operatorname{reg}\left(T_{\sigma}^{\prime}\right) .
$$

(f) Supposons que $\sigma=\sigma_{1} \cup \sigma_{2}$ avec $\sigma_{1} \cap \sigma_{2}=\varnothing$ alors

$$
P\left(\sigma_{1}\right) P\left(\sigma_{2}\right)=P\left(\sigma_{2}\right) P\left(\sigma_{1}\right)=0, \quad P(\sigma)=P\left(\sigma_{1}\right)+P\left(\sigma_{2}\right) .
$$

Soit maintenant $\Sigma$ l'ensemble des sous-ensembles finis de $\rho_{q \phi}^{s}(T)$ ordonné par inclusion. On vient de voir qu'à chaque $\sigma \in \Sigma$ on peut associer une projection spectrale généralisée $P(\sigma)$. D'après (f) il est clair que l'application $\sigma \mapsto P(\sigma)$ est un homomorphisme de treillis de $\Sigma$ dans l'ensemble des projections sur $H$.

Le théorème suivant se déduit de ce qu'on vient de voir et du Théorème 4.10 
Théorème 5.1. Soit $T \in B(H)$, (avec les notations précédentes) on a:

(i) L'application $\sigma \mapsto P(\sigma)$ est un homomorphisme de treillis de $\Sigma$ dans l'ensemble de projections sur $H$.

(ii) Si $H_{\sigma}=P(\sigma)(H)$ alors $\forall \sigma \in \Sigma, H_{\sigma}$ est invariant par $T$.

(iii) Si on note $\tilde{i}_{\sigma}$ la restriction de $T \grave{a} H_{\sigma}$ alors $\sigma\left(T_{\sigma}\right)=\sigma$.

(iv) Si $T_{\sigma}^{\prime}$ dénote la restriction de $T$ à $H_{\sigma}^{\prime}=(I-P(\sigma))(H)$ alors $\operatorname{reg}(T) \cup \sigma \subseteq$ $\operatorname{reg}\left(T_{\sigma}^{\prime}\right)$.

\section{REFERENCES}

1. G. R. Allan, On one-sided inverses in Banach algebras of holomorphic vector-valued functions, J. London Math. Soc. 42 (1967), 463-470.

2. __ Holomorphic vector-valued functions on a domain of holomorphy, J. London Math. Soc. 42 (1967), 509-513.

3. C. Apostol, The reduced minimum modulus, Michigan Math. J. 32 (1985), 279-294.

4. C. Apostol and K. Clancey, Generalized inverses and spectral theory, Trans. Amer. Math. Soc. 215 (1976), 293-300.

5. __ On generalized resolvants, Proc. Amer. Math. Soc. 58 (1976), 163-168.

6. T. Kato, Perturbation theory for nullity, deficiency, and other quantities of linear operators, J. Analyse Math. 6 (1958), 261-322.

7. J. Ph. Labrousse, Les opérateurs quasi-Fredholm: une généralisation des opérateurs semiFredholm, Rend. Circ. Mat. Palermo (2) 29 (1980), 161-258.

8. M. Mbekhta, Généralisation de la décomposition de Kato aux opérateurs paranormaux et spectraux, Thèse de 3ème cycle, Université de Nice, 1984.

9. __ Généralisation de la décomposition de Kato aux opérateurs paranormaux et spectraux, Glasgow Math. J. 29 (1987), 159-175.

10. __, Sur la théorie spectrale généralisée, C. R. Acad. Sci. Paris Sér. I 306 (1988), 593-596.

11. __ Résolvant généralisé et théorie spectrale, J. Operator Theory 21 (1989), 69-105.

12. P. Saphar, Contribution à l'étude des applications linéaires dans un espace de Banach, Bull. Soc. Math. France 92 (1964), 363-384.

Département de Mathematique, Université de Nice, 06034 Nice Cedex, France

E-mail address: blh@frmop11.bitnet

FRANCE 